\title{
Increased risk for tooth extraction in primary hyperparathyroidism and hypercalcemia: a population study
}

\author{
A. Koman ${ }^{1,2}$ (D) P. Näsman ${ }^{3}$ - A. Discacciati ${ }^{4}$ - A. Ekbom ${ }^{5}$ - I-L. Nilsson ${ }^{1,2} \cdot$ G. Sandborgh-Englund ${ }^{3,6}$
}

Received: 25 July 2019 / Accepted: 23 October 2019 / Published online: 2 December 2019

(C) The Author(s) 2019

\begin{abstract}
The aim of this study was to analyze dental comorbidities in untreated primary hyperparathyroidism (pHPT). Patients with pHPT subjected to parathyroidectomy (PTX) at Karolinska University Hospital, Stockholm, during 2011-2016 $(n=982)$ were selected from the Scandinavian Quality Register of Thyroid, Parathyroid and Adrenal surgery and compared to a general population cohort $(n=2944)$, matched for age and gender. Dental data was obtained from the Swedish Dental Health Registry for the 3 years prior to PTX. The incidence rate ratios (IRRs) of tooth loss by extraction, periodontal interventions, and dental visit rate were analyzed by Poisson regression models. In order to analyze the impact of disease severity, the PHPT cohort was sub-grouped based on preoperative serum levels of ionized calcium $\left(\mathrm{S}_{-} \mathrm{Ca}^{2+}\right)$. The total number of tooth extractions, periodontal interventions, and number of visits were similar in the cohorts. PHPT patients belonging to the quartile with the highest $\mathrm{S}-\mathrm{Ca}^{2+}(\geq 1.51 \mathrm{mmol} / \mathrm{L}) \mathrm{had}$ increased risk for tooth extraction (IRR $1.85 ; 95 \%$ CI 1.39-2.46). Female gender independently amplified the risk (IRR 1.341, $P<0.027$ ). This study indicates an association between pHPT and oral disorders reflected by increased tooth loss by extraction related to high $\mathrm{S}-\mathrm{Ca}^{2}$. Increased awareness of dental comorbidity in primary hyperparathyroidism may benefit a large group of patients with a common disease through earlier detection and prevention.
\end{abstract}

Keywords Dental care utilization $\cdot$ Tooth extraction $\cdot$ Periodontal $\cdot$ Hypercalcemia $\cdot$ Primary hyperparathyroidism $\cdot$ National register

Electronic supplementary material The online version of this article (https://doi.org/10.1007/s00784-019-03137-y) contains supplementary material, which is available to authorized users.

A. Koman

anna.koman@ki.se

1 Department of Endocrine Tumors and Sarcoma, Karolinska University Hospital, Stockholm, Sweden

2 Department of Molecular Medicine and Surgery, Karolinska Institutet, 17177 Stockholm, Sweden

3 Department of Dental Medicine, Karolinska Institutet, Stockholm, Sweden

4 Institute of Environmental Medicine, Karolinska Institutet, Stockholm, Sweden

5 Clinical Epidemiology Unit, Karolinska Institutet, Stockholm, Sweden

6 Academic Center for Geriatric Dentistry, Stockholm, Sweden

\section{Introduction}

Primary hyperparathyroidism (pHPT) is characterized by an excess secretion of parathyroid hormone (PTH) and an inappropriate elevation of ionized calcium $\left(\mathrm{S}-\mathrm{Ca}^{2+}\right)$ in blood, hypercalcemia. The diagnosis is easily determined by biochemical serum analysis. PTH is an 84 amino-acid hormone produced by the chief-cells in the parathyroid glands and constitutes the most important regulator of the free calcium $\left(\mathrm{S}_{-} \mathrm{Ca}^{2+}\right)$ homeostasis and plays a key role in bone remodeling [1].

The clinical presentation of $\mathrm{pHPT}$ has changed during the last century. Historically, pHPT was considered a rare disorder with notable morbidity, e.g., severe skeletal lesions and deformities due to demineralization and calcification of parenchymal organs, nephrolithiasis, and altered mental status caused by an extracellular excess of calcium [2]. Raised awareness of the pHPT diagnosis and more exact methods for measuring $\mathrm{S}_{-} \mathrm{Ca}^{2+}$ and PTH as well as the introduction of biochemical screening have contributed to a significant rise in incidence of pHPT. Today, $\mathrm{pHPT}$ is 
the third most common endocrine disorder, often diagnosed earlier and consequently, at a biochemically mild stage. The diagnosis is more prevalent in women and incidence increases with age. In the Swedish population, the prevalence of pHPT is estimated at $1 \%$, yet among postmenopausal women, it can be as high as $3-5 \%$ [3]. The cause is most often a benign parathyroid adenoma and the only curative treatment is surgical resectionparathyroidectomy (PTX). For patients with so-called asymptomatic pHPT, i.e., without obvious skeletal and kidney involvement, treatment is often delayed until complications occur. Guidelines regarding treatment and indications for surgery are continuously revised as the disease has become more prevalent [4].

Historically, osteitis fibrosa cystica was a classic complication of pHPT often associated with brown tumors of the jaw and pronounced periodontal lesions [5].

Knowledge regarding the impact and the clinical relevance of pHPT on oral health in the population today is limited. The association between $\mathrm{pHPT}$ and several known risk factors for dental disorders, e.g., osteoporosis, cardiovascular disease, diabetes, and psychiatric symptoms, is well-known [6] [7, 8]. However, there is little evidence of any association between early-stage pHPT and oral disorders. The current knowledge of the oral manifestations associated with pHPT is mainly based on historic data from case reports and limited case series. In a recent review summarizing the available literature between 1975 and 2016, including 205 articles and altogether 245 patients, a variety of symptoms and signs affecting oral health was reported. The findings were most commonly related to expansile bone effects and the second most common finding was oral pain. Frequent radiological findings were cortical destruction and tooth displacement [9]. Loss of the lamina dura, previously considered pathognomonic for pHPT, seemed to be one of the most common findings and was suggested to be related to increased bone turnover. Thus, the effect of pHPT on dental comorbidities is primarily hypothesized as being related to periodontal disease and ultimately, tooth loss. Extreme findings of pHPT-related brown tumors in the jaw, caused by microfractures and bleeding in an osteolytic lesion and ossifying fibromas, are today rarities and should raise suspicion for a genetic disorder known as the HPT-Jaw-tumor syndrome, caused by a defined mutation affecting the $C D C 73$ gene $[5,10]$.

\section{Molecular aspects and associations between parathyroid disorders and oral health}

PTH secretion is normally tightly regulated by a complex endocrine feedback loop involving calcium-sensing receptors (CaSR) at the surface of the parathyroid cells. PTH acts to increase the circulating $\mathrm{Ca}^{2+}$ concentration through three major pathways: by renal tubular reabsorption of calcium, by release of calcium from the bone mineral component, and by conversion of vitamin $\mathrm{D}$ to the active form, increasing the intestinal uptake of calcium.

PTH is the key regulator of bone remodeling. A continuous physiologic renewing of the skeletal tissue is essential to preserve a healthy bone. PTH normally exerts both catabolic and anabolic effects. In healthy individuals, bone absorption is followed by activation of osteoblasts and refilling of the degraded bone. In pHPT, the balanced bone turnover is disturbed leading to a catabolic state and a reversible reduced bone mineral density of both trabecular and cortical bone [11]. A recovered balanced bone turnover following surgical cure (PTX) enables refilling of the bone. Up to 1 year after PTX, the risk of fractures is increased; thereafter, the risk is equal to healthy controls [12].

In addition to the associations between disturbances in bone metabolism, pHPT and dental disorders share other common risk factors. Mental disorders including anxiety and depression are common in pHPT [13]. Based on a systematic review and metaanalysis, these disorders were associated with increased dental decay and tooth loss [14]. Furthermore, both pHPT and oral disorders are closely related to the occurrence and prognosis of cardiovascular disorders [15]. The exact mechanisms and possible impact of these associations are still not thoroughly researched.

\section{Objectives}

In this study, the intention was to analyze if the pattern of dental health care consumption in untreated $\mathrm{pHPT}$ patients differed from the background population. The hypothesis was that the deranged calcium metabolism and the altered bone remodeling, associated with $\mathrm{pHPT}$, might affect oral health with a subsequent impact on dental care consumption. The primary aim of this study was to analyze dental interventions prior to PTX in a pHPT-cohort in comparison to a matched cohort from the general population, focusing on tooth loss by extraction, interventions related to periodontal disease, and total number of dental care visits. A secondary aim was to explore the impact of the severity of hypercalcemia related to pHPT on tooth loss by extraction, interventions related to periodontal disease, and total number of dental care visits.

\section{Methods}

\section{Study design and setting}

This observational cohort study is based on data from regional and national Swedish registries. All Swedish residents are assigned a unique personal identity number, either at birth or upon immigration, which is used in all health and census registries and allows for linkage among national registries. The Swedish health care registries are enclosed by rigorous legal 
and ethical frameworks to ensure the integrity of the individual $[16,17]$. Ethical approval is required for scientific utilization of the Swedish registry data.

\section{Data sources}

The Scandinavian Quality Registry of Thyroid, Parathyroid and Adrenal surgery, SQRTPA, was founded in 2004. The registry covers about $95 \%$ of all PTX-surgeries performed in Sweden and contains information on preoperative work up, biochemical measures, preoperative imaging, complications after surgery, pathology reports, and lead times from the time of referral to 6 months postoperatively [18]. For registration in the SQRTPA, patient's informed consent is mandatory.

A pHPT patient cohort registered in SQRTPA that underwent curative PTX between January 2011 and December 2017 in Stockholm County was identified (Fig. 1). The patient cohort was compared to a reference cohort collected from the Total Population Register. This cohort was previously selected as a reference population for a study on head- and neck cancer and oral health [19]. The reference population was matched to the pHPT cohort on age ( \pm 1 year) and gender. (matching ratio 1:3).

The Swedish Dental Health Register (DHR) commenced in July 2008 and includes information on dental care under the National Dental Care Benefit Scheme [20].

\section{Information assessment}

Information on age at PTX, the level of free calcium in plasma $\left(\mathrm{S}-\mathrm{Ca}^{2+}\right)$, and the weight of the excised parathyroid adenoma was individually obtained from the regional server of SQRTPA for each member of the pHPT cohort.

Information on dental interventions was obtained from the DHR for both cohorts. Data on dental treatment, such as date, and type of treatment was obtained for a maximum time period of 3 years prior to index date (date of PTX) for all individuals included in the cohorts. The average time was 2.8 years.

\section{Outcomes}

The primary outcome was number of tooth extractions in patients with hypercalcemia related to $\mathrm{pHPT}$, compared to the reference cohort. Secondary endpoints were treatment of periodontal disease (including prevention, non-surgical and surgical treatment of periodontal disease; no. of interventions per year) and the total dental care consumption (no. of dental visits per year).

\section{Statistical analyses}

In order to account for the matched design of the study, fixedeffects Poisson regression models (conditioned on the matching strata) were used to estimate the incidence rate ratios
Fig. 1 Flow chart of the study procedure

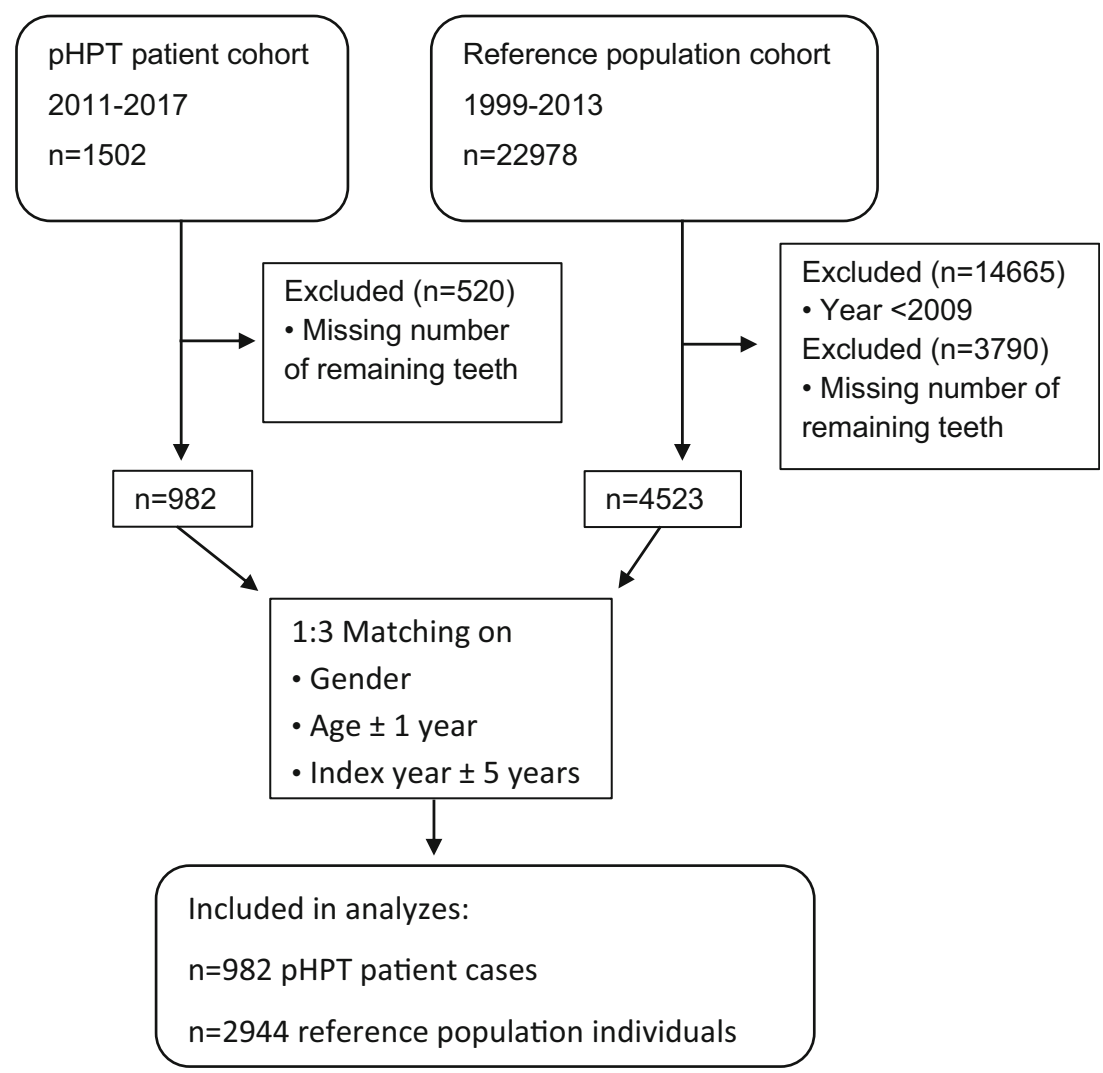


(IRRs) for tooth extractions, periodontal interventions, and total number of visits comparing the pHPT cohort to the reference cohort. In addition, the pHPT cohort was further categorized into quartiles based on the preoperative $\mathrm{S}-\mathrm{Ca}^{2+}$ (as a proxy for disease severity). IRRs for tooth extractions, periodontal interventions, and total number of visits were calculated for each S-Ca ${ }^{2+}$ quartile versus the reference cohort.

In a complementary sensitivity analysis restricted to the pHPT cohort only, Poisson regression models were used to estimate the IRRs for tooth extractions, periodontal interventions, and total number of visits related to a $1-g$ increase in weight of the excised parathyroid adenoma (serving as postoperative verifying proxy for severity and duration of disease) [21]. All models were adjusted for age, index year, and number of teeth. Time in the study was included as an offset variable after logarithmic transformation. Subjects with missing values on $\mathrm{S}-\mathrm{Ca}^{2+}(n=4)$ or on weight of the excised parathyroid adenoma $(n=43)$ were excluded from the analyses that involved those variables. All statistical analyses were performed using Stata (version 15, Stata Corp LP, College Station, TX).

The methods employed in this study are consistent with the STROBE (Strengthening the Reporting of Observational Studies in Epidemiology) guidelines for a human observational study (http://www.strobe-statement.org).

\section{Results}

The patient cohort included 982 pHPT patients (748 females) at a median age of 62 years with a preoperative median $\mathrm{S}-\mathrm{Ca}^{2+}$ of $1.45 \mathrm{mmol} / \mathrm{L}$. The reference cohort consisted of 2944 Stockholm County residents matched on age and gender. Characteristics are presented in Table 1.

There were no significant differences between the cohorts with respect to the incidence of the primary and secondary outcomes, e.g., tooth extractions, periodontal interventions, and total number of visits (Table 2).
Table 1 Descriptive characteristics of the patients with primary hyperparathyroidism and the reference population cohort

\begin{tabular}{|c|c|c|}
\hline Characteristic & PHPT cohort $(n=982)$ & Reference cohort $(n=2944)$ \\
\hline \multicolumn{3}{|l|}{ Age* at time for PTX } \\
\hline Median (IQ range) & $62(53,71)$ & $62(53,71)$ \\
\hline $20-39$ & $71(7.2 \%)$ & $211(7.2 \%)$ \\
\hline $40-59$ & $357(36.4 \%)$ & $1048(35.6 \%)$ \\
\hline $60-79$ & $479(48.8 \%)$ & $1450(49.3 \%)$ \\
\hline $80+$ & $75(7.6 \%)$ & $235(8.0 \%)$ \\
\hline \multicolumn{3}{|l|}{ Gender } \\
\hline Male & $234(23.8 \%)$ & $702(23.8 \%)$ \\
\hline Female & $748(76.2 \%)$ & $2242(76.2 \%)$ \\
\hline \multicolumn{3}{|l|}{ Calcium* before PTX } \\
\hline $\mathrm{S}-\mathrm{Ca}^{2+}(\mathrm{mmol} / \mathrm{L})$ & $1.45(1.41,1.51)$ & $\mathrm{n} / \mathrm{a}$ \\
\hline Total S-Ca $(\mathrm{mmol} / \mathrm{L})$ & $2.77(2.69,2.88)$ & $\mathrm{n} / \mathrm{a}$ \\
\hline \multicolumn{3}{|l|}{6 months postoperatively } \\
\hline $\mathrm{S}-\mathrm{Ca}^{2+}(\mathrm{mmol} / \mathrm{L})$ & $1.26(1.23,1.30)$ & $\mathrm{n} / \mathrm{a}$ \\
\hline Total S-Ca (mmol/L) & $2.40(2.35,2.48)$ & $\mathrm{n} / \mathrm{a}$ \\
\hline \multicolumn{3}{|l|}{ Dental interventions** } \\
\hline Tooth extractions & 367 & 827 \\
\hline Periodontal interventions $* * *$ & 2171 & 5438 \\
\hline Total number of visits & 6120 & 16,651 \\
\hline \multicolumn{3}{|l|}{ Remaining teeth* } \\
\hline Median (IQ range) & $28(25,29)$ & $27(25,29)$ \\
\hline $0-9$ & $31(3.2 \%)$ & $76(2.6 \%)$ \\
\hline 10 to 19 & $67(6.8 \%)$ & $207(7.0 \%)$ \\
\hline $20+$ & $884(90.0 \%)$ & $2661(90.4 \%)$ \\
\hline Weight excised parathyroid adenoma (gram) & $0.45(0.25,0.96)$ & $\mathrm{n} / \mathrm{a}$ \\
\hline
\end{tabular}

$P T X$, parathyroidectomy; $S-\mathrm{Ca}^{2+}$, serum ionized calcium mmol/L; total $S$-Ca, total serum calcium mmol/L

*Median (interquartile range), **dental interventions during 3 years prior to PTX, ***including preventive, nonsurgical, and surgical periodontal interventions 
Table 2 Over all comparison of dental care utilization in patients with $\mathrm{pHPT}$ compared to the reference population cohort*

\begin{tabular}{lllll}
\hline & $\begin{array}{l}\text { pHPT cohort }(n=982) \\
\text { Total number of events }\end{array}$ & $\begin{array}{l}\text { Reference cohort }(n=2944) \\
\text { Total number of events }\end{array}$ & IRR & $95 \%$ CI \\
\hline Tooth extraction & 367 & 827 & 1.103 & $(0.894-1.361)$ \\
Periodontal interventions & 2171 & 5438 & 0.954 & $(0.879-1.035)$ \\
Total number of visits & 6120 & 16,651 & 1.006 & $(0.961-1.053)$ \\
\hline
\end{tabular}

$p H P T$, primary hyperparathyroidism; $I R R$, incidence rate ratio

*Fixed-effect Poisson regression, further adjusted for age, index year, and number of teeth
The analysis showed that the pHPT patients with $\mathrm{S}-\mathrm{Ca}^{2+}$ in the upper (4th) quartile had a markedly increased incidence of tooth extraction (IRR 1.853, $P<0.001$ ) (Table 3).

Within the patient cohort, a lower number of natural teeth slightly predisposed less tooth extractions (IRR 0.924, $P<0.001)$. The number of dental care visits per year increased with age (IRR 1.013, $P<0.001)$. Female gender was a significant risk factor for tooth extractions (IRR 1.341, $P<0.027$ ). After correction for $\mathrm{S}_{-} \mathrm{Ca}^{2+}$, adenoma weight was an independent risk factor for tooth extractions (IRR 1.047, $P<0.030$ ) (Table 4).

\section{Discussion}

The aim of this introductory, registry-based study was to compare dental interventions and dental care utilization among pHPT patients to a cohort of the normal population. Overall, no differences in utilized dental care were observed when comparing the cohorts. However, a sub-group analysis revealed differences related to the preoperative $\mathrm{S}-\mathrm{Ca}^{2+}$ level. The group of patients with the highest preoperative calcium levels had a significantly increased risk of more drastic dental intervention, i.e., tooth extractions. The observed association between higher $\mathrm{S}-\mathrm{Ca}^{2+}$ and increased tooth loss by extraction indicates an association between disease severity and oral disorders. The coherent, independent relationship between the risk of tooth extractions and the weight of the removed parathyroid adenoma as a proxy for more severe disease further strengthens this assumption. It is important to recognize that the majority patients treated for $\mathrm{pHPT}$ in developed countries today are detected with mild hypercalcemia. The median calcium levels in the fourth quartile of this patient cohort still are considered only moderately elevated.

Periodontitis is a major cause of tooth loss in adults [6]. Emerging evidence indicates an association between osteoporosis/osteopenia and risk of periodontal attachment loss [22, 23]. The bone is the main target of PTH, and pHPT is associated with abnormal bone remodeling [24]. Pronounced periodontal lesions are predominantly observed in advanced stages of pHPT. Our findings of a correlation between severity of hypercalcemia and an increased risk of tooth loss by extraction are consistent with previous knowledge [9].
Table 3 Comparison of dental care utilization related to severity of hypercalcemia prior PTX vs the reference population cohort*

\begin{tabular}{|c|c|c|c|c|}
\hline & Total number of events & IRR & $95 \% \mathrm{CI}$ & $p$ \\
\hline \multicolumn{5}{|c|}{$\mathrm{Q} 1\left(\mathrm{~S}-\mathrm{Ca}^{2+} 1.19-1.41 \mathrm{mmol} / \mathrm{L}\right)$} \\
\hline Tooth extraction & 65 & 0.785 & $(0.569-1.083)$ & 0.104 \\
\hline Periodontal interventions & 705 & 1.045 & $(0.937-1.165)$ & 0.429 \\
\hline Total number of visits & 1844 & 1.016 & $(0.954-1.083)$ & 0.614 \\
\hline \multicolumn{5}{|c|}{ Q2 $\left(\mathrm{S}^{-\mathrm{Ca}^{2+}} 1.42-1.45 \mathrm{mmol} / \mathrm{L}\right)$} \\
\hline Tooth extraction & 69 & 0.881 & $(0.619-1.256)$ & 0.484 \\
\hline Periodontal interventions & 409 & 0.784 & $(0.689-0.893)$ & $<0.001$ \\
\hline Total number of visits & 1337 & 0.988 & $(0.919-1.062)$ & 0.745 \\
\hline \multicolumn{5}{|c|}{ Q3 $\left(\mathrm{S}-\mathrm{Ca}^{2+} 1.46-1.51 \mathrm{mmol} / \mathrm{L}\right)$} \\
\hline Tooth extraction & 68 & 0.934 & $(0.675-1.291)$ & 0.679 \\
\hline Periodontal interventions & 532 & 0.925 & $(0.821-1.043)$ & 0.204 \\
\hline Total number of visits & 1439 & 0.963 & $(0.898-1.032)$ & 0.285 \\
\hline \multicolumn{5}{|c|}{$\mathrm{Q} 4\left(\mathrm{~S}^{-\mathrm{Ca}^{2+}} 1.52-2.62 \mathrm{mmol} / \mathrm{L}\right)$} \\
\hline Tooth extraction & 162 & 1.853 & $(1.395-2.462)$ & $<0.001$ \\
\hline Periodontal interventions & 517 & 1.036 & $(0.914-1.174)$ & 0.579 \\
\hline Total number of visits & 1476 & 1.065 & $(0.991-1.144)$ & 0.086 \\
\hline
\end{tabular}

$P T X$, parathyroidectomy; $S-C a^{2+}$, serum ionized calcium; $Q$, quartile; $I R R$, incidence rate ratio

*Fixed-effect Poisson regression, further adjusted for age, index year and number of teeth 
Table 4 Independent risk factors for dental interventions in patients with primary hyperparathyroidism, $n=936$. Adjusted for S-Ca ${ }^{2+*}$

\begin{tabular}{lllr}
\hline & IRR & $95 \%$ CI & $p$ \\
\hline Female gender & & & \\
$\quad$ Tooth extraction & 1.341 & $(1.034-1.739)$ & 0.027 \\
Periodontal interventions & 0.928 & $(0.837-1.028)$ & 0.153 \\
Total number of visits & 1.007 & $(0.946-1.072)$ & 0.816 \\
Age & & & \\
Tooth extraction & 1.003 & $(0.994-1.011)$ & 0.556 \\
Periodontal interventions & 1.015 & $(1.011-1.019)$ & $<0.001$ \\
Total number of visits & 1.013 & $(1.010-1.015)$ & $<0.001$ \\
Remaining teeth & & & \\
Tooth extraction & 0.924 & $(0.913-0.916)$ & $<0.001$ \\
Periodontal interventions & 1.006 & $(0.998-1.015)$ & 0.159 \\
Total number of visits & 1.000 & $(0.995-1.004)$ & 0.914 \\
Adenoma weight (gram) & & & \\
Tooth extraction & 1.047 & $(1.004-1.091)$ & 0.003 \\
Periodontal interventions & 1.004 & $(0.964-1.046)$ & 0.738 \\
Total number of visits & 0.994 & $(0.979-1.010)$ & 0.472 \\
\hline
\end{tabular}

$P T X$, parathyroidectomy; $S-\mathrm{Ca}^{2+}$, serum ionized calcium; $Q$, quartile; $I R R$, incidence rate ratio

*Fixed-effect Poisson regression, further adjusted for age, index year, and number of teeth

Furthermore, the analyses indicated an amplified increased risk for tooth loss associated with female gender and increasing age in the pHPT cohort compared to the reference population, independent of calcium-levels. In the context of pHPT being a common disease more prevalent in women and with an incidence that increases with age, these results are important to highlight. Interestingly, an altered pattern of dental care utilization was also observed in mild hypercalcemia as compared to the reference population (Table 3; Table 4). Further studies are warranted in regard to mild disease.

The present study has obvious limitations. The study design does not permit analyses of causal relationships. The observation time was restricted to a maximum of 3 years prior to PTX, which is a relatively short time span for long-term complications to develop. In addition, the results may be affected by the frequency of dental health care visits. Moreover, detailed data on symptoms and indications for PTX was not available. The included laboratory data is limited to $\mathrm{S}-\mathrm{Ca}^{2+}$ and does not allow any conclusions concerning other possible biochemical mechanisms. Comorbidities such as osteoporosis, cardiovascular disorders, and diabetes, all linked to risk of periodontal disease, are more prevalent in $\mathrm{PHPT}$ which might bias he results $[6,25,26]$.

The strength of this study is the size of the study population and the validity of the information available in the registries. The Swedish dental health care is subsidized and both publicly and privately run dental offices are registered and therefore the register covers nearly the entire population [27].

Primary hyperparathyroidism is considered a common disease and yet, the impacts of oral manifestations in pHPT remain a relatively unexplored area of research. In future studies, relevant comorbidities, socioeconomic variables, and drug exposure will be included in a national population study.

\section{Conclusions}

This study provides new and relevant perspectives in a sparsely studied field, at potential benefit for a large group of patients with a common disease. Increased multidisciplinary awareness of oral comorbidity in pHPT may present many advantageous effects by earlier detection and prevention.

Acknowledgments The authors are grateful to all colleagues, co-workers, and patients for providing data for this study. All co-writers contributed to the concept and design of the study and to intellectual perspectives of the content of the manuscript.

Funding Information Open access funding provided by Karolinska Institutet.

\section{Compliance with ethical standards}

Conflict of interest The authors declare that they have no conflict of interest.

Ethical approval Ethical approval was obtained by Regional Ethical Committee in Stockholm (Dnr 2017/192-32 and 2017/2-12).

Informed consent Informed consent was not deemed necessary since this observational cohort study was based on previously collected data from regional and national Swedish registries.

Open Access This article is distributed under the terms of the Creative Commons Attribution 4.0 International License (http:// creativecommons.org/licenses/by/4.0/), which permits unrestricted use, distribution, and reproduction in any medium, provided you give appropriate credit to the original author(s) and the source, provide a link to the Creative Commons license, and indicate if changes were made.

\section{References}

1. Mackenzie-Feder J, Sirrs S, Anderson D, Sharif J, Khan A (2011) Primary hyperparathyroidism: an overview. Int J Endocrinol 2011: 251410. https://doi.org/10.1155/2011/251410

2. Khan AA, Hanley DA, Rizzoli R, Bollerslev J, Young JE, Rejnmark L, Thakker R, D'Amour P, Paul T, Van Uum S, Shrayyef MZ, Goltzman D, Kaiser S, Cusano NE, Bouillon R, Mosekilde L, Kung AW, Rao SD, Bhadada SK, Clarke BL, Liu J, Duh Q, Lewiecki EM, Bandeira F, Eastell R, Marcocci C, Silverberg SJ, Udelsman R, Davison KS, Potts JT Jr, Brandi ML, Bilezikian JP (2017) Primary hyperparathyroidism: review and 
recommendations on evaluation, diagnosis, and management. A Canadian and international consensus. Osteoporos Int 28:1-19. https://doi.org/10.1007/s00198-016-3716-2

3. Siilin H, Rastad J, Ljunggren O, Lundgren E (2008) Disturbances of calcium homeostasis consistent with mild primary hyperparathyroidism in premenopausal women and associated morbidity. J Clin Endocrinol Metab 93:47-53. https://doi.org/10.1210/jc.2007-0600

4. Wilhelm SM, Wang TS, Ruan DT, Lee JA, Asa SL, Duh QY, Doherty GM, Herrera MF, Pasieka JL, Perrier ND, Silverberg SJ, Solorzano CC, Sturgeon C, Tublin ME, Udelsman R, Carty SE (2016) The American Association of Endocrine Surgeons Guidelines for Definitive Management of Primary Hyperparathyroidism. JAMA Surg 151:959-968. https://doi.org/ 10.1001/jamasurg.2016.2310

5. Silverman S Jr, Ware WH, Gillooly C Jr (1968) Dental aspects of hyperparathyroidism. Oral Surg Oral Med Oral Pathol 26:184-189

6. Pihlstrom BL, Michalowicz BS, Johnson NW (2005) Periodontal diseases. Lancet (London, England) 366:1809-20. https://doi.org/ 10.1016/S0140-6736(05)67728-8

7. Nilsson IL (2018) Primary hyperparathyroidism: should surgery be performed on all patients? Current evidence and residual uncertainties J Intern Med doi. https://doi.org/10.1111/joim.12840

8. Meurman JH, Sanz M, Janket SJ (2004) Oral health, atherosclerosis, and cardiovascular disease. Crit Rev Oral Biol Med 15:403-413

9. Palla B, Burian E, Fliefel R, Otto S (2018) Systematic review of oral manifestations related to hyperparathyroidism. Clin Oral Investig 22:1-27. https://doi.org/10.1007/s00784-017-2124-0

10. Misiorowski W, Czajka-Oraniec I, Kochman M, Zgliczynski W, Bilezikian JP (2017) Osteitis fibrosa cystica-a forgotten radiological feature of primary hyperparathyroidism. Endocrine 58:380-385. https://doi.org/10.1007/s12020-017-1414-2

11. Rolighed L, Rejnmark L and Christiansen P (2014) Bone involvement in primary hyperparathyroidism and changes after parathyroidectomy. Eur Endocrinol 10:84-87. doi: https://doi.org/10. 17925/EE.2014.10.01.84

12. Vestergaard P, Mollerup CL, Frokjaer VG, Christiansen P, BlichertToft M, Mosekilde L (2000) Cohort study of risk of fracture before and after surgery for primary hyperparathyroidism. BMJ (Clinical research ed) 321:598-602. https://doi.org/10.1136/bmj.321.7261. 598

13. Bilezikian JP, Bandeira L, Khan A, Cusano NE (2018) Hyperparathyroidism. Lancet (London, England) 391:168-178. https://doi.org/10.1016/S0140-6736(17)31430-7

14. Kisely S, Sawyer E, Siskind D, Lalloo R (2016) The oral health of people with anxiety and depressive disorders - a systematic review and meta-analysis. J Affect Disord 200:119-132. https://doi.org/10. 1016/j.jad.2016.04.040

15. Yu YH, Chasman DI, Buring JE, Rose L, Ridker PM (2015) Cardiovascular risks associated with incident and prevalent periodontal disease. J Clin Periodontol 42:21-28. https://doi.org/10. $1111 /$ jcpe. 12335

16. Council tSR. the Swedish Research Council. www. registerforskning.se. Accessed Acces Date
17. Ludvigsson JF, Haberg SE, Knudsen GP, Lafolie P, Zoega H, Sarkkola C, von Kraemer S, Weiderpass E, Norgaard M (2015) Ethical aspects of registry-based research in the Nordic countries. Clin Epidemiol 7:491-508. https://doi.org/10.2147/CLEP.S90589

18. The Scandinavian Quality Register of Thyroid, Parathyroid and Adrenal surgery. The Scandinavian Quality Register of Thyroid, Parathyroid and Adrenal surgery. http://www.thyroidparathyroidsurgery.com/. Accessed Acces Date

19. Lexomboon D, Karlsson P, Adolfsson J, Ekbom A, Naimi-Akbar A, Bahmanyar S, Montgomery S, Sandborgh-Englund G (2017) Consumption and direct costs of dental care for patients with head and neck cancer: a 16-year cohort study. PLoS One 12:e182877. https://doi.org/10.1371/journal.pone.0182877

20. Socialstyrelsen, Tandhalsosregistret. The National Board of Health and Welfare (Socialstyrelsen), Ministry of Health and Social Affairs. http://www.socialstyrelsen.se/register/halsodataregister/ tandhalsoregistret. Accessed Acces Date 2019

21. Moretz WH 3rd, Watts TL, Virgin FW Jr, Chin E, Gourin CG, Terris DJ (2007) Correlation of intraoperative parathyroid hormone levels with parathyroid gland size. Laryngoscope 117:1957-1960. https://doi.org/10.1097/MLG.0b013e31813c14fc

22. Penoni DC, Fidalgo TK, Torres SR, Varela VM, Masterson D, Leao AT, Maia LC (2017) Bone density and clinical periodontal attachment in postmenopausal women: a systematic review and metaanalysis. J Dent Res 96:261-269. https://doi.org/10.1177/ 0022034516682017

23. Yoshihara A, Seida Y, Hanada N, Miyazaki H (2004) A longitudinal study of the relationship between periodontal disease and bone mineral density in community-dwelling older adults. J Clin Periodontol 31:680-684. https://doi.org/10.1111/j.1600-051X. 2004.00548.x

24. Silva BC, Bilezikian JP (2015) Parathyroid hormone: anabolic and catabolic actions on the skeleton. Curr Opin Pharmacol 22:41-50. https://doi.org/10.1016/j.coph.2015.03.005

25. Liljestrand JM, Havulinna AS, Paju S, Mannisto S, Salomaa V, Pussinen PJ (2015) Missing teeth predict incident cardiovascular events, diabetes, and death. J Dent Res 94:1055-1062. https://doi. org $/ 10.1177 / 0022034515586352$

26. D'Aiuto F, Gkranias N, Bhowruth D, Khan T, Orlandi M, Suvan J, Masi S, Tsakos G, Hurel S, Hingorani AD, Donos N, Deanfield JE and Group T (2018) Systemic effects of periodontitis treatment in patients with type 2 diabetes: a 12 month, single-centre, investigator-masked, randomised trial. Lancet Diabetes Endocrinol 6:954 965. doi: https://doi.org/10.1016/S2213-8587(18)30038-X

27. Ljung R, Lundgren F, Appelquist M, Cederlund A (2019) The Swedish dental health register - validation study of remaining and intact teeth. BMC Oral Health 19:116. https://doi.org/10.1186/ s12903-019-0804-7

Publisher's note Springer Nature remains neutral with regard to jurisdictional claims in published maps and institutional affiliations. 